\title{
A conjecture of Biggs concerning the resistance of a distance-regular graph
}

\author{
Greg Markowsky \\ gmarkowsky@gmail.com \\ Pohang Mathematics Institute \\ POSTECH \\ Pohang, 790-784 \\ Republic of Korea
}

\author{
Jacobus Koolen \\ jacobus_koolen@yahoo.com \\ Department of Mathematics \\ POSTECH \\ Pohang, 790-784 \\ Republic of Korea
}

Submitted: Apr 12, 2010; Accepted: May 18, 2010; Published: May 25, 2010

Mathematics Subject Classification: 05E30

\begin{abstract}
Biggs conjectured that the resistance between any two points on a distanceregular graph of valency greater than 2 is bounded by twice the resistance between adjacent points. We prove this conjecture, give the sharp constant for the inequality, and display the graphs for which the conjecture most nearly fails. Some necessary background material is included, as well as some consequences.
\end{abstract}

\section{Introduction}

The main goal of this paper is to prove the following conjecture of Biggs:

Theorem 1 Let $G$ be a distance-regular graph with degree larger than 2 and diameter $D$. If $d_{j}$ is the electric resistance between any two vertices of distance $j$, then

$$
\max _{j} d_{j}=d_{D} \leqslant K d_{1}
$$

where $K=1+\frac{94}{101} \approx 1.931$. Equality holds only in the case of the Biggs-Smith graph.

We remark that for degree 2 the theorem is trivially false. This theorem implies several statements concerning random walks on distance-regular graphs, which will be given at the end of the paper. General background material on the concept of electric resistance, as well as its connection to random walks, can be found in the excellent references [6] and [2]. Biggs' conjecture originally appeared in [1], which discusses electric resistance on distance-regular graphs only. To understand the proof of the conjecture, one must 
understand much of the material in [1]. We have therefore decided to include the material from [1] which is key to Theorem 1. This appears in Section 3, following the relevant graph-theoretic definitions in Section 2. Section 4 gives our proof of the theorem, and Section 5 gives some consequences, including several in the field of random walks.

\section{Distance-regular graphs}

All the graphs considered in this paper are finite, undirected and simple (for unexplained terminology and more details, see for example [4]). Let $G$ be a connected graph and let $V=V(G)$ be the vertex set of $G$. The distance $d(x, y)$ between any two vertices $x, y$ of $G$ is the length of a shortest path between $x$ and $y$ in $G$. The diameter of $G$ is the maximal distance occurring in $G$ and we will denote this by $D=D(G)$. For a vertex $x \in V(G)$, define $K_{i}(x)$ to be the set of vertices which are at distance $i$ from $x(0 \leqslant i \leqslant D)$ where $D:=\max \{d(x, y) \mid x, y \in V(G)\}$ is the diameter of $G$. In addition, define $K_{-1}(x):=\emptyset$ and $K_{D+1}(x):=\emptyset$. We write $x \sim_{G} y$ or simply $x \sim y$ if two vertices $x$ and $y$ are adjacent in $G$. A connected graph $G$ with diameter $D$ is called distance-regular if there are integers $b_{i}, c_{i}(0 \leqslant i \leqslant D)$ such that for any two vertices $x, y \in V(G)$ with $d(x, y)=i$, there are precisely $c_{i}$ neighbors of $y$ in $K_{i-1}(x)$ and $b_{i}$ neighbors of $y$ in $K_{i+1}(x)$ (cf. [4, p.126]). In particular, distance-regular graph $G$ is regular with valency $k:=b_{0}$ and we define

$a_{i}:=k-b_{i}-c_{i}$ for notational convenience. The numbers $a_{i}, b_{i}$ and $c_{i}(0 \leqslant i \leqslant D)$ are called the intersection numbers of $G$. Note that $b_{D}=c_{0}=a_{0}=0, b_{0}=k$ and $c_{1}=1$. The intersection numbers of a distance-regular graph $G$ with diameter $D$ and valency $k$ satisfy (cf. [4, Proposition 4.1.6])

(i) $k=b_{0}>b_{1} \geqslant \cdots \geqslant b_{D-1}$;

(ii) $1=c_{1} \leqslant c_{2} \leqslant \cdots \leqslant c_{D}$;

(iii) $b_{i} \geqslant c_{j}$ if $i+j \leqslant D$.

Moreover, if we fix a vertex $x$ of $G$, then $\left|K_{i}\right|$ does not depend on the choice of $x$ as $c_{i+1}\left|K_{i+1}\right|=b_{i}\left|K_{i}\right|$ holds for $i=1,2, \ldots D-1$. In the next section, it will be shown that the resistance between any two vertices of $G$ can be calculated explicitly using only the intersection array, so that the proof can be conducted using only the known properties of the array.

\section{$3 \quad$ Electric resistance on distance-regular graphs}

Henceforth let $G$ be a distance-regular graph with $n$ vertices, degree $k \geqslant 3$, and diameter $D$. Let $V=V(G)$ and $E=E(G)$ be the vertex and edge sets, respectively, of $G$. To calculate the resistance between any two vertices we use Ohm's Law, which states that

$$
V=I R
$$


where $V$ represents a difference in voltage(or potential), $I$ represents current, and $R$ represents resistance. That is, we imagine that our graph is a circuit where each edge is a wire with resistance 1 . We attach a battery of voltage $V$ to two distinct vertices $u$ and $v$, producing a current through the graph. The resistance between the $u$ and $v$ is then $V$ divided by the current produced. The current flowing through the circuit can be determined by calculating the voltage at each point on the graph, then summing the currents flowing from $u$, say, to all vertices adjacent to $u$. Calculating the voltage at each point is thereby seen to be an important problem. A function $f$ on $V$ is harmonic at a point $z \in V$ if $f(z)$ is the average of neighboring values of $f$, that is

$$
\sum_{x \sim z}(f(x)-f(z))=0
$$

The voltage function on $V$ can be characterized as the unique function which is harmonic on $V-\{u, v\}$ having the prescribed values on $u$ and $v$. For our purposes, on the distanceregular graph $G$, we will first suppose that $u$ and $v$ are adjacent. It is easy to see that, for any vertex $z,|d(u, z)-d(v, z)| \leqslant 1$, where $d$ denotes the ordinary graph-theoretic distance. Thus, any $z$ must be contained in a unique set of one of the following forms:

$$
\begin{aligned}
& K_{i}^{i}=\{x: d(u, x)=i \text { and } d(v, x)=i\} \\
& K_{i}^{i+1}=\{x: d(u, x)=i+1 \text { and } d(v, x)=i\} \\
& K_{i+1}^{i}=\{x: d(u, x)=i \text { and } d(v, x)=i+1\}
\end{aligned}
$$

Suppose that $\left(b_{0}, b_{1}, \ldots, b_{D-1} ; c_{1}, c_{2}, \ldots, c_{D}\right)$ is the intersection array of $G$. For $0 \leqslant i \leqslant$ $D-1$ define the numbers $\phi_{i}$ recursively by

$$
\begin{aligned}
\phi_{0} & =n-1 \\
\phi_{i} & =\frac{c_{i} \phi_{i-1}-k}{b_{i}}
\end{aligned}
$$

We then have the following fundamental proposition.

Proposition 1 The function $f$ defined on $V$ by

$$
\begin{aligned}
& f(u)=-f(v)=\phi_{0} \\
& f(z)=0 \text { for } x \in K_{i}^{i} \\
& f(z)=\phi_{i} \text { for } x \in K_{i+1}^{i} \\
& f(z)=-\phi_{i} \text { for } x \in K_{i}^{i+1}
\end{aligned}
$$

is harmonic on $V-\{u, v\}$.

In the following intersection diagram, the value of $f$ on each set is given directly outside the set. 


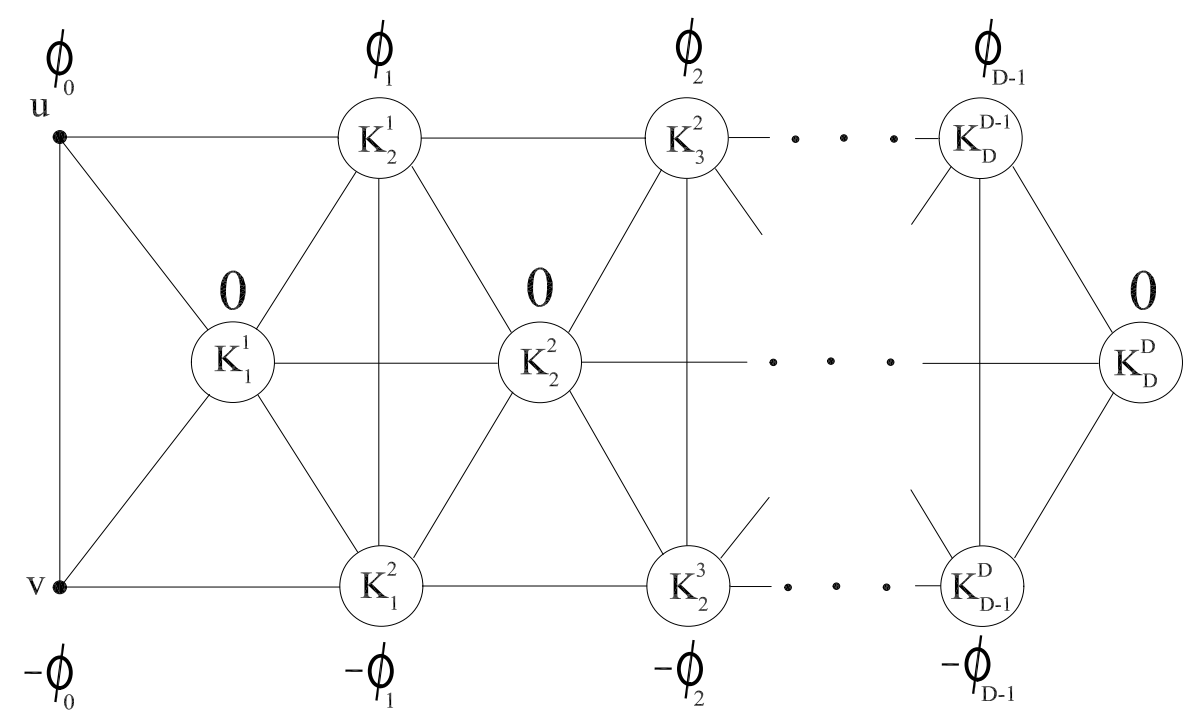

Figure 1

To prove Proposition 1 we need the following lemma, which may be of interest in its own right.

Lemma 1 Let $z \in G$, and let $K_{i}=\{x: d(z, x)=i\}$ as in Section 2. Let $e_{i}$ be the number of edges of $G$ with one endpoint in $K_{i}$ and the other in $K_{i+1}$. Then

$$
\phi_{i}=\frac{k \sum_{j>i}\left|K_{j}\right|}{e_{i}}
$$

Proof: Since $\phi_{0}=n-1=\sum_{j>0}\left|K_{j}\right|$ and $e_{0}=k$, it is clear that (7) holds for $i=0$. We need therefore only verify that the numbers $\psi_{i}=\frac{k \sum_{j>i}\left|K_{j}\right|}{e_{i}}$ satisfy the recursive relation given in (5). This is immediate from the facts that $e_{i}=b_{i}\left|K_{i}\right|$ and $e_{i-1}=c_{i}\left|K_{i}\right|$, for we see that

$$
\begin{aligned}
\frac{c_{i} \psi_{i-1}-k}{b_{i}} & =\frac{c_{i}\left(\frac{k\left|K_{i}\right|+k \sum_{j>i}\left|K_{j}\right|}{e_{i-1}}\right)-k}{b_{i}} \\
& =\frac{c_{i} k \sum_{j>i}\left|K_{j}\right|}{b_{i} e_{i-1}} \\
& =\frac{k \sum_{j>i}\left|K_{j}\right|}{b_{i} K_{i}} \\
& =\frac{k \sum_{j>i}\left|K_{j}\right|}{e_{i}} \\
& =\psi_{i}
\end{aligned}
$$


Proof of Proposition 1: Suppose first that $z \in K_{i}^{i}$ for some $i$. The points adjacent to $z$ must lie within $K_{i}^{i} \bigcup K_{i-1}^{i-1} \bigcup K_{i+1}^{i+1} \bigcup K_{i}^{i+1} \bigcup K_{i}^{i-1} \cup K_{i-1}^{i} \cup K_{i+1}^{i}$. Since $b_{i}$ is equal to the number of adjacent points in $K_{i+1}^{i+1} \bigcup K_{i}^{i+1}$, and also in the set $K_{i+1}^{i+1} \bigcup K_{i+1}^{i}$, we see that

$$
\mid\left\{x: z \sim x \text { and } x \in K_{i+1}^{i}\right\}|=|\left\{x: z \sim x \text { and } x \in K_{i}^{i+1}\right\} \mid
$$

A similar argument shows

$$
\mid\left\{x: z \sim x \text { and } x \in K_{i-1}^{i}\right\}|=|\left\{x: z \sim x \text { and } x \in K_{i}^{i-1}\right\} \mid
$$

It follows from this that

$$
\sum_{x \sim z} f(x)=0=f(z)
$$

and $f$ is harmonic at $z$. Now suppose that $z \in K_{i+1}^{i}$ with $1 \leqslant i \leqslant D-2$. Here the points adjacent to $z$ must lie within $K_{i+1}^{i} \bigcup K_{i}^{i-1} \bigcup K_{i+2}^{i+1} \bigcup K_{i}^{i} \bigcup K_{i+1}^{i+1} \bigcup K_{i}^{i+1}$. The number of edges from $z$ to points in $K_{i}^{i-1}$ is $c_{i}$ and to points in $K_{i+2}^{i+1}$ is $b_{i+1}$. Let the number of edges from $z$ to points in $K_{i}^{i+1}$ be $\alpha$. Then the number of edges from $z$ to other points in $K_{i+1}^{i}$ is given by $k+\alpha-c_{i+1}-b_{i}$. We therefore have

$$
\begin{aligned}
\sum_{x \sim z} f(x) & =b_{i+1} \phi_{i+1}+c_{i} \phi_{i-1}+\left(k+\alpha-c_{i+1}-b_{i}\right) \phi_{i}+\alpha\left(-\phi_{i}\right) \\
& =k \phi_{i}=k f(z)
\end{aligned}
$$

where we have used the following equations equivalent to the recursive relation in (5).

$$
\begin{aligned}
& c_{i} \phi_{i-1}=b_{i} \phi_{i}+k \\
& b_{i+1} \phi_{i+1}=c_{i+1} \phi_{i}-k
\end{aligned}
$$

We see that $f$ is harmonic at $z$. The same argument works for $z \in K_{D}^{D-1}$, except that there is some difficulty in using the last equation in (13), as $b_{D}=0$, and $\phi_{i}$ was only defined for $i \leqslant D-1$. Happily, Lemma 1 solves our dilemma, for as an immediate consequence we obtain $\phi_{D-1}=\frac{k}{c_{D}}$. Thus, defining $\phi_{D}=0$ is consistent with (13), and $f$ is harmonic on $K_{D}^{D-1}$. By symmetry, $f$ is harmonic at all points lying in sets of the form $K_{i+1}^{i}$, and the proof is complete.

Corollary 1. $\phi_{i}>\phi_{i+1}$ for $0 \leqslant i \leqslant D-2$

Proof: Suppose $\phi_{i} \leqslant \phi_{i+1}$ for some $i$. Due to the monotonicity of the sequences $b_{i}, c_{i}$, we would have

$$
\phi_{i+2}=\frac{c_{i+2} \phi_{i+1}-k}{b_{i+2}} \geqslant \frac{c_{i+1} \phi_{i}-k}{b_{i+1}}=\phi_{i+1}
$$

Continuing in this way we would have $\phi_{D-1} \geqslant \phi_{D-2}$. On the other hand, by harmonicity $\phi_{D-1}$ is the weighted average of the values $\phi_{D-2}, 0$, and $-\phi_{D-1}$, so that $\phi_{D-1}<\phi_{D-2}$. This is a contradiction. 
It may interest the reader to note that the subtracted constant $k$ in the numerator of the recursive relation of (5) can be replaced by any constant without affecting harmonicity outside of the sets $K_{D}^{D-1}$ and $K_{D-1}^{D}$. However, $k$ is the only constant which gives $\phi_{D}=0$, and therefore is the constant dictated by the requirement that $f$ be harmonic and attain the boundary values of $(n-1)$ and $-(n-1)$ at $u$ and $v$. The resistance between $u$ and $v$ can now easily be computed as the voltage difference between the points, $2 \phi_{0}=2(n-1)$, divided by the current $I$ flowing through the circuit. This current is the sum of the voltage differences between $u$ and vertices adjacent to $u$, and is readily computable as $I=n k$. We see that the resistance between $u$ and $v$ is

$$
R_{u v}=\frac{2(n-1)}{n k}=\frac{n-1}{m}
$$

where $m=n k / 2$ is the number of edges in $G$. This result is in fact an immediate consequence of Foster's Network Theorem(see [2] or [7]), and was derived, among other things, by other methods in [10]. In the remainder of this section, however, it will be more conceptually convenient to keep $I$ and the $\phi$ 's in the formulas rather than their explicit values, as this reminds us that they represent the current and voltages, respectively. Calculating the resistances between nonadjacent vertices might now seem to be a formidable task, but in fact there is virtually no more to be done. We have the following proposition.

Proposition 2 The resistance between two vertices of distance $j$ in a graph is given by

$$
\frac{2 \sum_{0 \leqslant i<j} \phi_{i}}{I}
$$

Proof: Suppose $d(u, v)=j$. We can choose points $x_{0}=u, x_{1}, \ldots, x_{j}=v$ such that $x_{i} \sim x_{i+1}$. For any pair of adjacent points $y, z$ we let $f_{y z}$ be the unique function on $V$ given in Proposition 1 which is harmonic on $V-\{y, z\}$ and which satisfies $f(w)=-f(z)=\phi_{0}$. The key claim is that for any three points $w, y, z$ with $y \sim w \sim z$ the function $f_{y w}+f_{w z}$ is harmonic on $V-\{y, z\}$. This is clear for all points in $V-\{y, z\}$ except $w$. To show harmonicity at $w$, note that a current of $I$ flows into $w$ due to $f_{y w}$, whereas a current of $I$ flows out of $w$ due to $f_{w z}$. The net current flow into $w$ is therefore 0 , which is equivalent to harmonicity(see [6]). Thus, the voltage function $g=\sum_{0 \leqslant i \leqslant j-1} f_{x_{i} x_{i+1}}$, which is harmonic on $V-\{u, v\}$, gives rise to a current of $I$ flowing from $u$ to $v$. We must therefore calculate the values of the function $g$ at the points $u$ and $v$. It is straightforward to verify that $f_{x_{i} x_{i+1}}(u)=\phi_{i}$ (since $u$ lies in the set $K_{i+1}^{i}$ formed with respect to the pair $\left.x_{i}, x_{i+1}\right)$, and likewise $f_{x_{i} x_{i+1}}(v)=-\phi_{D-(i+1)}$. Thus, $g(u)=\sum_{0 \leqslant i<j} \phi_{i}$ and $g(v)=-\sum_{0 \leqslant i<j} \phi_{i}$. The result follows.

\section{Proof of Theorem}

In fact, we will prove a statement stronger than Theorem 1 . Let $\mathcal{E}$ be the set of the following four graphs, with corresponding properties listed: 


\begin{tabular}{lccr} 
Name $^{1}$ & Vertices & Intersection array & $\frac{\phi_{1}+\ldots+\phi_{D-1}}{\phi_{0}}$ \\
\hline Biggs-Smith Graph & 102 & $(3,2,2,2,1,1,1 ; 1,1,1,1,1,1,3)$ & 0.930693 \\
Foster Graph & 90 & $(3,2,2,2,2,1,1,1 ; 1,1,1,1,2,2,2,3)$ & 0.896067 \\
Flag graph of GH(2,2) & 189 & $(4,2,2,2,2,2 ; 1,1,1,1,1,2)$ & 0.882979 \\
Tutte's 12-Cage & 126 & $(3,2,2,2,2,2 ; 1,1,1,1,1,3)$ & 0.872
\end{tabular}

Theorem 2 Other than graphs in $\mathcal{E}$, for any distance regular graph with degree at least 3 we have

$$
\phi_{1}+\ldots+\phi_{D-1}<.87 \phi_{0}
$$

This clearly implies Theorem 1 and shows that the graphs in $\mathcal{E}$ are the extremal cases.

Proof of Theorem 2: The proof proceeds by considering a number of separate cases, and leans heavily on the standard reference [4]. Without access to this book, the proof will likely be incomprehensible to the reader. In the estimates used in the proof, the $-k$ in the numerator of the recurrence relation is largely ignored, but the reader should be warned that this term is by no means unnecessary. That is because it is crucial that the $\phi_{i}$ 's form a monotone decreasing sequence, and without the $-k$ this would not be the case. Nevertheless, we will from this point forth mainly use the facts $\phi_{i}<\frac{c_{i} \phi_{i-1}}{b_{i}}$ and $\phi_{i}<\phi_{i-1}$. We are required to show

$$
\frac{\phi_{1}+\ldots+\phi_{D-1}}{\phi_{0}} \leqslant .87
$$

for all graphs not in $\mathcal{E}$.

Case $1: D=2$.

We need only show $\phi_{1}<.87 \phi_{0}$. This is clear if $b_{1}>1$, since $c_{1}=1$ and $\phi_{i}<\frac{c_{i} \phi_{i-1}}{b_{i}}$. The case $b_{1}=1$ is known to occur only in the case of the Cocktail party graphs, and it is simple to verify the relation in this case.

Case $2: k=3$.

It is known(see [4], Theorem 7.5.1) that the only distance-regular graphs of degree 3 with diameter greater than 2 are given by the intersection arrays below, and which give rise to the resistances given:

\footnotetext{
${ }^{1}$ The referee has pointed out that Tutte's 12-Cage may be more accurately referred to as Benson's graph, and indeed the literature is mixed on this point. The referee further remarked that the Flag graph of $G H(2,2)$ can also be realized as the line graph of Tutte's 12-Cage, or Benson's graph. In this table, we are employing the names given in [4].
} 


\begin{tabular}{lccr} 
Name & Vertices & Intersection array & $\frac{\phi_{1}+\ldots \phi_{D-1}}{\phi_{0}}$ \\
\hline Cube & 8 & $(3,2,1 ; 1,2,3)$ & 0.428571 \\
Heawood graph & 14 & $(3,2,2 ; 1,1,3)$ & 0.461538 \\
Pappus graph & 18 & $(3,2,2,1 ; 1,1,2,3)$ & 0.588235 \\
Coxeter graph & 28 & $(3,2,2,1 ; 1,1,1,2)$ & 0.666667 \\
Tutte's 8-cage & 30 & $(3,2,2,2 ; 1,1,1,3)$ & 0.655172 \\
Dodecahedron & 20 & $(3,2,1,1,1 ; 1,1,1,2,3)$ & 0.842105 \\
Desargues graph & 20 & $(3,2,2,1,1 ; 1,1,2,2,3)$ & 0.710526 \\
Tutte's 12-cage & 126 & $(3,2,2,2,2,2 ; 1,1,1,1,1,3)$ & 0.872 \\
Biggs-Smith graph & 102 & $(3,2,2,2,1,1,1 ; 1,1,1,1,1,1,3)$ & 0.930693 \\
Foster graph & 90 & $(3,2,2,2,2,1,1,1 ; 1,1,1,1,2,2,2,3)$ & 0.896067
\end{tabular}

Case $3: k=4$.

It is known(see [3]) that the only distance-regular graphs of degree 4 with diameter greater than 2 are given by the intersection arrays below, and which give rise to the resistances given:

\begin{tabular}{lccr} 
Name & Vertices & Intersection array & $\frac{\phi_{1}+\ldots \phi_{D-1}}{\phi_{0}}$ \\
\hline$K_{5,5}$ minus a matching & 10 & $(4,3,1 ; 1,3,4)$ & 0.296296 \\
Nonincidence graph of $P G(2,2)$ & 14 & $(4,3,2 ; 1,2,4)$ & 0.307692 \\
Line graph of Petersen graph & 15 & $(4,2,1 ; 1,1,4)$ & 0.428571 \\
4-cube & 16 & $(4,3,2,1 ; 1,2,3,4)$ & 0.422222 \\
Flag graph of $P G(2,2)$ & 21 & $(4,2,2 ; 1,1,2)$ & 0.5 \\
Incidence graph of $P G(2,3)$ & 26 & $(4,3,3 ; 1,1,4)$ & 0.32 \\
Incidence graph of $A G(2,4)$-p.c. & 32 & $(4,3,3,1 ; 1,1,3,4)$ & 0.376344 \\
Odd graph $O_{4}$ & 35 & $(4,3,3 ; 1,1,2)$ & 0.352941 \\
Flag graph of $G Q(2,2)$ & 45 & $(4,2,2,2 ; 1,1,1,2)$ & 0.681818 \\
Doubled odd graph & 70 & $(4,3,3,2,2,1,1 ; 1,1,2,2,3,3,4)$ & 0.521739 \\
Incidence graph of $G Q(3,3)$ & 80 & $(4,3,3,3 ; 1,1,1,4)$ & 0.417722 \\
Flag graph of $G H(2,2)$ & 189 & $(4,2,2,2,2,2 ; 1,1,1,1,1,2)$ & 0.882979 \\
Incidence graph of $G H(3,3)$ & 728 & $(4,3,3,3,3,3 ; 1,1,1,1,1,4)$ & 0.485557
\end{tabular}

Case $4: D \leqslant 5, b_{1} \geqslant 5$.

This case was done initially by Biggs in [1], without the restriction on $b_{1}$ but with the constant 1 in place of .87. Nevertheless, when we restrict $b_{1}$ as above this is trivial, because $\frac{\phi_{1}}{\phi_{0}}<\frac{1}{b_{1}}$ and $\phi_{i} \leqslant \phi_{1}$ for all $i>0$. Therefore,

$$
\frac{\phi_{1}+\ldots+\phi_{D-1}}{\phi_{0}} \leqslant \frac{(D-1) \phi_{1}}{\phi_{0}} \leqslant \frac{4}{b_{1}} \leqslant .8
$$

Henceforth, in all cases for which $b_{1} \geqslant 5$ we can assume $D \geqslant 6$. In what follows, let $j$ denote the smallest value such that $c_{j} \geqslant b_{j}$. If $c_{j}>b_{j}$, then, since $c_{D-j} \leqslant b_{j}$ and the $c_{i}$ 's are nondecreasing, we see that $D-j<j$, hence $D \leqslant 2 j-1$. If $c_{j}=b_{j}$, then it follows 
from Corollary 5.9.6 of [4] that $c_{2 j}>b_{2 j}$. For this to occur, either $c_{2 j}>b_{j}$ or $c_{j}>b_{2 j}$. By the same argument as before, we obtain $D \leqslant 3 j-1$. This will be of fundamental importance in our proof. To begin with, we see that when $D \geqslant 6$ we must have $j \geqslant 3$.

Case 5: $G$ is a line graph.

The distance-regular line graphs have been classified, and appear in Theorem 4.2.16 of [4]. All such graphs with $k \geqslant 3$ have $D \leqslant 2$ and are therefore covered by Case 1 , with two exceptions. First of all, $G$ may be a generalized $2 D$-gon of order $(1, s)$. The intersection array of $G$ is then of the form $\left(2\left(a_{1}+1\right), a_{1}+1, \ldots, a_{1}+1 ; 1,1, \ldots, 1,2\right)$, with $a_{1}>1$. The other possibility is that $G$ could be the line graph of a Moore graph, and in this case the intersection array of $G$ is of the form $(2 \kappa-2, \kappa-1, \kappa-2 ; 1,1,4)$, for some $\kappa \geqslant 3$. In both of these cases it is straightforward to verify that the conclusion of the theorem holds.

Case $6: b_{1} \geqslant 5, j=3, c_{2}=1$.

Since $j=3, b_{2} \geqslant 2$ and $D \leqslant 8$. We have

$$
\frac{\phi_{1}+\ldots+\phi_{D-1}}{\phi_{0}} \leqslant \frac{\phi_{1}+6 \phi_{2}}{\phi_{0}} \leqslant \frac{1}{b_{1}}+\frac{6}{2 b_{1}}=\frac{4}{b_{1}} \leqslant .8
$$

Case $7: b_{1} \geqslant 5, j=3, c_{2}>1$.

By Theorem 5.4.1 in [4], $c_{2} \leqslant \frac{2}{3} c_{3}$. If $c_{3}>b_{3}$ then $D \leqslant 2 j-1=5$, which was covered in Case 4. If $c_{3}=b_{3} \leqslant b_{2}$, then if we assume $\frac{c_{2}}{b_{2}} \leqslant \frac{1}{2}$ we have

$$
\frac{\phi_{1}+\ldots+\phi_{D-1}}{\phi_{0}} \leqslant \frac{\phi_{1}+6 \phi_{2}}{\phi_{0}} \leqslant \frac{1}{b_{1}}+\frac{3}{b_{1}}=\frac{4}{b_{1}} \leqslant .8
$$

On the other hand, if it is not the case that $\frac{c_{2}}{b_{2}} \leqslant \frac{1}{2}$, then the proof of Theorem 5.4.1 of [4] implies that $G$ contains a quadrangle. By Corollary 5.2 .2 in [4], $D \leqslant \frac{2 k}{k+1-b_{1}}$. It is straightforward to verify that the fact that $k \geqslant b_{1}+1$ implies that

$$
\frac{2 k}{k+1-b_{1}} \leqslant b_{1}+1
$$

We therefore see that the fact that $G$ contains a quadrangle implies $D \leqslant b_{1}+1$. Furthermore, we still have $\frac{c_{2}}{b_{2}} \leqslant \frac{2}{3}$ by Theorem 5.4.1 of [4]. We therefore have

$$
\frac{\phi_{1}+\ldots+\phi_{D-1}}{\phi_{0}} \leqslant \frac{\phi_{1}+\left(b_{1}-1\right) \phi_{2}}{\phi_{0}} \leqslant \frac{1}{b_{1}}+\frac{2\left(b_{1}-1\right)}{3 b_{1}}=\frac{2 b_{1}+1}{3 b_{1}} \leqslant .7
$$

Case 8: $b_{1} \geqslant 5, j \geqslant 4, c_{2}=1$.

If $j \geqslant 4$ and $b_{2}=2$ then we must have $b_{3}=2, c_{3}=1$, so that $\frac{b_{2} b_{3}}{c_{2} c_{3}}=4$. On the other hand, if this does not occur than $\frac{b_{2}}{c_{2}} \geqslant 3$. We will consider these cases separately. 
Subcase 1: $\frac{b_{2}}{c_{2}} \geqslant 3$.

For $i<j$ we have $b_{1} \geqslant b_{i}>c_{i}$, and for any $i$ with $c_{i}>1$ we must have $b_{i}<b_{1}$, by Proposition 5.4.4 in [4]. Thus, $\frac{c_{i}}{b_{i}} \leqslant \frac{b_{1}-2}{b_{1}-1}$. Define $\alpha=\frac{b_{1}-2}{b_{1}-1}$. We have

$$
\frac{\phi_{1}+\ldots+\phi_{D-1}}{\phi_{0}} \leqslant \frac{1}{b_{1}}+\frac{1}{3 b_{1}}+\frac{\alpha}{3 b_{1}}+\ldots+\frac{\alpha^{j-3}}{3 b_{1}}+\frac{(2 j-1) \alpha^{j-3}}{3 b_{1}}
$$

Replace the second through $(j-1)$ th term by a geometric series to obtain

$$
\begin{aligned}
\frac{\phi_{1}+\ldots+\phi_{D-1}}{\phi_{0}}<\frac{1}{b_{1}} & +\frac{1}{3 b_{1}}\left(\frac{1}{1-\frac{b_{1}-2}{b_{1}-1}}\right)+\frac{(2 j-1) \alpha^{j-3}}{3 b_{1}} \\
& <\frac{1}{b_{1}}+\frac{b_{1}-1}{3 b_{1}}+\frac{2(j-1 / 2) \alpha^{j-1 / 2}}{3 b_{1} \alpha^{5 / 2}}
\end{aligned}
$$

Simple calculus shows that the maximum of the function $u \alpha^{u}$ is $\frac{-1}{e \ln \alpha}$. We therefore obtain

$$
\frac{\phi_{1}+\ldots+\phi_{D-1}}{\phi_{0}}<\frac{b_{1}+2}{3 b_{1}}+\frac{-2}{3 b_{1}\left(\frac{b_{1}-2}{b_{1}-1}\right)^{5 / 2} e \ln \left(\frac{b_{1}-2}{b_{1}-1}\right)}
$$

It is straightforward to verify that the function $(b-2) \ln \left(\frac{b-2}{b-1}\right)$ is increasing in $b$, so that the right hand side of (26) achieves its maximum on the allowed range when $b_{1}=5$. Plugging in $b_{1}=5$ gives approximately .851 as a bound for (26).

Subcase 2: $\frac{b_{2} b_{3}}{c_{2} c_{3}} \geqslant 4$.

This follows much as in the previous case, except that we may simplify by using the slightly weaker bound $\frac{c_{i}}{b_{i}} \leqslant \frac{b_{1}-1}{b_{1}}$ for $i<j$. Let $\alpha=\frac{b_{1}-1}{b_{1}}$. Since $b_{2} \geqslant b_{3}$ and $c_{2} \leqslant c_{3}$ we must have $\frac{b_{2}}{c_{2}} \geqslant 2$. We then have

$$
\frac{\phi_{1}+\ldots+\phi_{D-1}}{\phi_{0}} \leqslant \frac{1}{b_{1}}+\frac{1}{2 b_{1}}+\frac{1}{4 b_{1}}+\frac{\alpha}{4 b_{1}}+\ldots+\frac{\alpha^{j-3}}{4 b_{1}}+\frac{(2 j-1) \alpha^{j-3}}{4 b_{1}}
$$

Following the steps in (31) above, we obtain

$$
\frac{\phi_{1}+\ldots+\phi_{D-1}}{\phi_{0}}<\frac{3}{2 b_{1}}+\frac{1}{4}+\frac{-1}{2 b_{1}\left(\frac{b_{1}-1}{b_{1}}\right)^{5 / 2} e \ln \left(\frac{b_{1}-1}{b_{1}}\right)}
$$

Again this is decreasing in $b_{1}$, and plugging in $b_{1}=5$ gives a bound for (28) of about 84 .

Case $9: b_{1} \geqslant 3, j \geqslant 4, c_{2}>1, G$ contains a quadrangle.

As in the argument given in Case 7 , we see that $G$ containing a quadrangle implies $D \leqslant b_{1}+1$. Furthermore, Theorem 5.4 .1 of [4] implies that $c_{3} \geqslant(3 / 2) c_{2}$. Since $j \geqslant 4$ and thus $b_{2} \geqslant b_{3}>c_{3}$ we must have $\frac{c_{2}}{b_{2}} \leqslant \frac{2}{3}$. This gives

$$
\frac{\phi_{1}+\ldots+\phi_{D-1}}{\phi_{0}} \leqslant \frac{1}{b_{1}}+\left(b_{1}-1\right) \frac{2}{3 b_{1}}=\frac{2 b_{1}+1}{3 b_{1}}
$$


When $b_{1} \geqslant 3$ this is bounded by .8 .

Case $10: b_{1} \geqslant 3, j \geqslant 4, c_{2} \geqslant 1, G$ does not contain a quadrangle.

In this case $G$ is a Terwilliger graph. By Corollary 1.16.6 of [4], if $k<50\left(c_{2}-1\right)$ then $D \leqslant 4$ and $b_{1} \geqslant 5$, which was covered in Case 4 . Thus, we can assume $k \geqslant 50\left(c_{2}-1\right)$, which implies $b_{1} \geqslant 10 c_{2}$. If $b_{2} \geqslant 3 c_{2}$ then we can follow the proof of Subcase 1 of Case 8 to obtain our result, so we may assume $b_{2} \leqslant 3 c_{2}$, which implies $b_{2}<\frac{b}{2}$. It follows from this that for $i<j \frac{c_{2}}{b_{2}} \leqslant \frac{\left(b_{1} / 2\right)-1}{b_{1} / 2}=\frac{b_{1}-2}{b_{1}}$. We set $\alpha=\frac{b_{1}-2}{b_{1}}$. By the proof of Theorem 5.4.1 in [4] we have $c_{3} \geqslant 2 c_{2}$. Since $b_{2} \geqslant b_{3}>c_{3} \geqslant 2 c_{2}$ we have $\frac{b_{2}}{c_{2}} \geqslant 2$. We compute

$$
\frac{\phi_{1}+\ldots+\phi_{D-1}}{\phi_{0}} \leqslant \frac{1}{b_{1}}+\frac{1}{2 b_{1}}+\frac{\alpha}{2 b_{1}}+\ldots+\frac{\alpha^{j-3}}{2 b_{1}}+\frac{(2 j-1) \alpha^{j-3}}{2 b_{1}}
$$

Replace the second through $(j-1)$ th term by a geometric series to obtain

$$
\begin{aligned}
\frac{\phi_{1}+\ldots \phi_{D-1}}{\phi_{0}}<\frac{1}{b_{1}}+\frac{1}{2 b_{1}} & \left(\frac{1}{1-\frac{b_{1}-2}{b_{1}}}\right)+\frac{(2 j-1) \alpha^{j-3}}{2 b_{1}} \\
& <\frac{1}{b_{1}}+\frac{1}{4}+\frac{(j-1 / 2) \alpha^{j-1 / 2}}{b_{1} \alpha^{5 / 2}}
\end{aligned}
$$

The maximum of the function $u \alpha^{u}$ is $\frac{-1}{e \ln \alpha}$. We therefore obtain

$$
\frac{\phi_{1}+\ldots \phi_{D-1}}{\phi_{0}}<\frac{1}{b_{1}}+\frac{1}{4}+\frac{-1}{b_{1}\left(\frac{b_{1}-2}{b_{1}}\right)^{5 / 2} e \ln \left(\frac{b_{1}-2}{b_{1}}\right)}
$$

As before, the function $(b-2) \ln \left(\frac{b-2}{b}\right)$ is increasing in $b$, so the right hand side of $(32)$ is decreasing in $b_{1}$. Plugging in $b_{1}=10$ (recall that $b_{1} \geqslant 10 c_{2} \geqslant 10$ ) gives approximately .64 as a bound.

Case $11 b_{1}=3$ or $4, k \geqslant 5, c_{2}=1$.

This will be broken down into cases by degree $k$. Proposition 1.2.1 in [4] implies that $\left(a_{1}+1\right) \mid k$, so since $b_{1}=k-a_{1}-1$ and $b_{1}>0$ we see that $b_{1} \geqslant k / 2$. This implies $k \leqslant 8$.

Subcase $k=8: b_{1}=3$ is ruled out because $\left(a_{1}+1\right) \mid k$. Suppose $b_{1}=4$. By Proposition 4.3.4 of [4], $G$ is a line graph, and is therefore covered by Case 5 .

Subcase $k=7$ : Since $\left(a_{1}+1\right) \mid k$, we must have $a_{1}=0$ and thus $b_{1}=6$, which is a contradiction.

Subcase $k=6$ : Since $\left(a_{1}+1\right) \mid k$, we have $a_{1} \in\{0,1,2\}$. If $a_{1}=0$, then $b_{1}=5$, a contradiction. If $a_{1}=1$, then as was shown in [9] $G$ is one of the following graphs.

\begin{tabular}{lccc} 
Name & Vertices & Intersection array & $\frac{\phi_{1}+\ldots \phi_{D-1}}{\phi_{0}}$ \\
\hline Colinearity graph of $G Q(2,2)$ & 15 & $(6,4 ; 1,3)$ & 0.142857 \\
Colinearity graph of $G H(2,2)$ & 27 & $(6,4,2 ; 1,2,3)$ & 0.269231 \\
Hamming graph $H(3,3)$ & 63 & $(6,4,4 ; 1,1,3)$ & 0.258065 \\
Halved Foster graph & 45 & $(6,4,2,1 ; 1,1,4,6)$ & 0.278409
\end{tabular}


If $a_{1}=2$, then by Proposition 4.3.4 of [4], $G$ is a line graph, and is therefore covered by Case 5.

Subcase $k=5$ : Since $\left(a_{1}+1\right) \mid k$, we must have $a_{1}=0$ and $b_{1}=4$. Suppose first that $b_{2}=3$ or 4 . Note that, for $i<j, \frac{c_{i}}{b_{i}} \leqslant \frac{2}{3}$, since $c_{i}+b_{i} \leqslant 5$. Using the same technique as in many of the previous cases we have

$$
\begin{aligned}
& \frac{\phi_{1}+\ldots \phi_{D-1}}{\phi_{0}}<\frac{1}{4}+\frac{1}{12}+\frac{1}{12}\left(\frac{2}{3}+\ldots+\left(\frac{2}{3}\right)^{j-2}\right)+\frac{1}{12}\left(\frac{2}{3}\right)^{j-2}(2 j-1) \\
& <\frac{1}{4}+\frac{1}{12}+\frac{3}{12}+\frac{1}{16}\left(\frac{2}{3}\right)^{j-2}(2 j-1)
\end{aligned}
$$

It is straightforward to verify that the last expression in (33) is decreasing in $j$ for $j \geqslant 3$. Plugging in $j=3$ gives a bound of $31 / 36<.87$. It remains only to consider $b_{2} \leqslant 2$. Suppose $b_{2}=2$. If $c_{3}=1$, it would follow from Corollary 4.3.12(ii) that 3 divides 20 . Thus, we can assume $c_{3} \geqslant 2$, and therefore $j=3$ and $D \leqslant 8$. We will first show that $n \leqslant 140$. Fix a point $u$ in $G$ and let $k_{i}=|\{v: d(u, v)=i\}|$. The numbers $k_{i}$ are easily computable through the intersection arrays by $k_{i}=\frac{\prod_{l=0}^{i-1} b_{i}}{\prod_{l=1}^{i} c_{i}}$. The $k_{i}$ 's are nonincreasing for $i \geqslant j$, so since $k_{3}=20$, if $D \leqslant 7$ we have $n \leqslant 1+5+6(20)<140$. Suppose $D=8$. Then $c_{6} \leqslant b_{2}=2$, so $c_{6}=2$ and this implies $b_{6}=1$. In this case, $k_{7}=10$, and thus $k_{8} \leqslant 10$ as well. We get $n \leqslant 1+5+5(20)+2(10)<140$ again. Since $k=5$, we get $k>(n-1) / 28$. Let $\theta=\left|\left\{i: b_{i}=c_{i}=2\right\}\right|$. If $\theta=3$, the maximal allowed value, we have the following calculations:

$$
\begin{aligned}
& \phi_{0}=n-1, \phi_{1}<\frac{n-1}{4}, \phi_{2}<\frac{n-1}{8}, \\
& \phi_{3}<\frac{2((n-1) / 8)-(n-1) / 28}{2}=\frac{6(n-1)}{56}, \\
& \phi_{4}<\frac{2(6(n-1) / 56)-(n-1) / 28}{2}=\frac{5(n-1)}{56}, \\
& \phi_{5}<\frac{2(5(n-1) / 56)-(n-1) / 28}{2}=\frac{4(n-1)}{56}
\end{aligned}
$$

Since $\phi_{6}, \phi_{7}<\phi_{5}$ we get

$$
\frac{\phi_{1}+\ldots \phi_{D-1}}{\phi_{0}}<\frac{1}{4}+\frac{1}{8}+\frac{6}{56}+\frac{5}{56}+3\left(\frac{4}{56}\right)=\frac{44}{56}<.87
$$

Similar but easier calculations handle the cases $\theta=2,1,0$. The case $b_{2}=1$ can also be handled in a similar way. Note that in this case $j=2$, so $D \leqslant 5$. If $D \leqslant 4$, then

$$
\frac{\phi_{1}+\ldots \phi_{D-1}}{\phi_{0}} \leqslant \frac{3 \phi_{1}}{\phi_{0}}<\frac{3}{4}
$$

If $D=5$, then $k_{1}=5, k_{2}=20$, and $k_{i} \leqslant 20$ for $i \geqslant j$ (since the $k_{i}$ 's are nonincreasing for $i \geqslant j$ ). It follows that $n \leqslant 86$, and therefore $k>\frac{n-1}{20}$. Furthermore, $c_{3} \leqslant b_{2}=1$, so 
$c_{3}=1$. Thus,

$$
\begin{aligned}
& \phi_{0}=n-1, \phi_{1}<\frac{(n-1)-k}{4} \leqslant \frac{19(n-1)}{80}, \\
& \phi_{2}<\frac{c_{2} \phi_{1}-k}{1}<\frac{15(n-1)}{80}, \\
& \phi_{3}, \phi_{4}<\frac{c_{2} \phi_{2}-k}{1}<\frac{11(n-1)}{80}
\end{aligned}
$$

And so

$$
\frac{\phi_{1}+\ldots \phi_{4}}{\phi_{0}}<\frac{19}{80}+\frac{15}{80}+2\left(\frac{11}{80}\right)=56 / 80=.7
$$

\section{Consequences}

As indicated in [1], there are some immediate consequences for random walks. Let $u$ be a vertex of $G$, and and suppose we start a random walk at $u$. For any other point $v$, we let the expected number of steps needed to hit $v$ be denoted $H_{u v}$. This is referred to as the hitting time. The commute time $C_{u v}$ is the expected number of steps necessary for the random walk to travel from $u$ to $v$ and back to $v$, and in the case of distance regular graphs is equal to $2 H_{u} v$. By Theorem 1 in [5], the expected commute time of a random walk between two points $u$ and $v$ is equal to $2 m R_{u v}$. Thus, from Theorem 1 in this paper, and the calculation of resistance given in Section 2, in a distance-regular graph with valency greater than 2 we have

\section{Proposition 3}

$$
\begin{aligned}
& H_{u v} \leqslant 2 m\left(\frac{n-1}{m}\right)=2(n-1) \\
& C_{u v} \leqslant 4 m\left(\frac{n-1}{m}\right)=4(n-1)
\end{aligned}
$$

The cover time $\operatorname{Co}(G)$ is the expected number of steps that our random walk requires before it has visited every site on $G$. Applying Theorem 3 in [5], we have

Proposition 4 For $n$ large,

$$
C o(G) \leqslant(4+o(1))(n-1) \ln n
$$

In fact, in [8] it was shown that for all graphs, distance-regular or otherwise, we have

$$
C o(G) \geqslant(1+o(1)) n \ln n
$$

so that the bound in Proposition 4 is the best possible, up to the multiplicative constant. Let $\sigma$ be the smallest nonzero eigenvalue of the Laplacian matrix. Note that $k-\sigma$ is the second largest eigenvalue of the adjacency matrix. Let $R_{\max }$ denote the largest resistance between points in $G$, which we have seen necessarily occurs when the points are at distance $D$. Combining Theorem 1 in this paper with Theorem 7 in [5], we have 


\section{Proposition 5}

$$
\sigma \geqslant \frac{1}{n R_{\max }} \geqslant \frac{m}{2 n(n-1)}=\frac{k}{4(n-1)}
$$

There have been discussions between the two authors as to whether Theorem 1 really gives new information on the structure of distance-regular graphs. It can be shown that any sequence of non-increasing $b_{i}$ 's and non-decreasing $c_{i}$ 's give rise to a sequence of potentials $\phi_{i}$, and that the $\phi_{i}$ 's are decreasing and remain positive. In that sense, a graph doesn't need to actually exist for a given intersection array in order for the potentials to be defined and behave correctly. Furthermore, any intersection arrays which can be ruled out as corresponding to actual graphs by this theorem could in theory be ruled out by the many facts from which we deduced the theorem. Nevertheless, this theorem does perhaps capture a large number of disparate and complicated results on distance-regular graphs in a simple statement. As an example, Theorem 2 shows that the following intersection arrays cannot be realized.

\begin{tabular}{lcr} 
Intersection array & Vertices & $\frac{\phi_{1}+\ldots \phi_{D-1}}{\phi_{0}}$ \\
\hline$(3,2,2,1,1,1,1 ; 1,1,1,1,1,1,3)$ & 62 & 1.04918 \\
$(5,2,2,1,1,1,1 ; 1,1,1,1,1,1,4)$ & 101 & 1.0375 \\
$(8,3,3,3,3,3,3,3,2,2,1 ; 1,2,2,3,3,3,3,3,3,3,8)$ & 150 & 0.938852
\end{tabular}

This can be shown by other methods, but the methods may differ between the examples, and may have much in common with the given proof of Theorem 2 in certain cases. Note that these intersection arrays satisfy a number of basic feasibility requirements, such as being monotone and having $c_{i} \leqslant b_{D-i}$ for all $i$. Note further that none of these arrays can be ruled out by Ivanov's bound(Corollary 5.9.6 of [4]). We therefore have hopes that this theorem can be found useful in the study of distance-regular graphs, both for disallowing certain intersection arrays and as a tool for proving other statements.

\section{Acknowledgements}

The first author was supported by Priority Research Centers Program through the National Research Foundation of Korea (NRF) funded by the Ministry of Education, Science and Technology (Grant \#2009-0094070). The second author was partially supported by the Basic Science Research Program through the National Research Foundation of Korea(NRF) funded by the Ministry of Education, Science and Technology (Grant \# 20090089826). 


\section{References}

[1] Biggs, N. (1993) Potential theory on distance-regular graphs. Combinatorics, Probability and Computing 2, p. 243-255.

[2] Biggs, N. (1997) Algebraic Potential Theory on Graphs, Bulletin of the London Mathematical Society, Volume 29, Number 6, p. 641-682.

[3] Brouwer, A., Koolen, J. (1999) The Distance-Regular Graphs of Valency Four, Journal of Algebraic Combinatorics, Volume 10, Issue 1, p. 5-24.

[4] Brouwer, A., Cohen, A., Neumaier, A. (1989) Distance Regular Graphs, SpringerVerlag.

[5] Chandra, A., Raghaven, P., Ruzzo, W., Smolensky, R., Tiwari, P. (1989) The electrical resistance of a graph captures its commute and cover time, Proc. 21st ACM STOC, pp. 574-586.

[6] Doyle, P. and Snell, J. (1984) Random Walks and Electric Networks, Carus Mathematical Monographs

[7] Foster, R. M. (1949) The Average Impedance of an Electrical Network, Contributions to Applied Mechanics (Reissner Anniversary Volume), pp. 333-340.

[8] Feige, U. (1995) A tight lower bound on the cover time for random walks on graphs, Random Struct. Alg., v. 6, pp. 433-438.

[9] Hiraki, A., Nomura, K., Suzuki, H. (2000) Distance-Regular Graphs of Valency 6 and $a_{1}=1$, Journal of Algebraic Combinatorics, v.11 n.2, p.101-134.

[10] Jafarizadeh, M. A., Sufiani, R., Jafarizadeh, S. (2009) Recursive calculation of effective resistances in distance-regular networks based on Bose-Mesner algebra and Christoffel-Darboux identity, J. Math. Phys. 50. 